\title{
Eventos adversos medicamentosos en pacientes ambulatorios
}

\author{
Adverse drug events in outpatients
}

Budnitz D et al. JAMA 2006 Oct 18; 296:1858-1866

\author{
Objetivo \\ Describir la frecuencia y características de los eventos adver- \\ sos medicamentosos (EAM) que conducen a consultas de \\ urgencia.
}

\section{Diseño \\ Estudio Multicéntrico Descriptivo.}

\section{Lugar y población}

Población ambulatoria de 63 hospitales de EEUU.

\section{Medición de resultados}

Se utilizaron datos registrados (enero de 2004 a diciembre de 2005) por un programa nacional de vigilancia de daño (Vigilancia Cooperativa de Efectos Adversos de Drogas, en inglés: CADES). Los datos fueron tomados de las historias clínicas de pacientes que visitaron las centrales de emergencia (CE) de las instituciones participantes. Se midieron y analizaron las visitas motivadas por EAM. Fueron considerados medicamentos los fármacos de venta libre y bajo receta, las vacunas, los suplementos dietarios y las vitaminas.

\section{Resultados principales}

Se notificaron 21.298 visitas a CE originadas por EAM, lo que implica una estimación anual ponderada de 701.547 pacientes afectados y una incidencia poblacional de 2,4 (IC95\%:1,7 a
$3,0)$ visitas por cada mil habitantes por año en todo el país. De estos casos, el 16,7\% (IC95\%: 13,1 a 20,3) requirió hospitalización. Los mayores de 65 años fueron más susceptibles a tener EAM que los menores (4,9 contra 2 por cada mil habitantes por año). Las visitas relacionadas a EAM constituyeron el 2,5\% (IC95\%: 2,0 a 3,1) del total de visitas por daños no intencionales a CE. De acuerdo al tipo de EAM, 53,4, 31 y $11,3 \%$ fueron clasificados como sobredosis no intencionales, reacciones alérgicas y efectos adversos respectivamente. Los medicamentos implicados con mayor frecuencia en eventos adversos fueron: insulinas ( $8 \%$ del total) warfarina $(6,2 \%)$ amoxicilina $(4,3 \%)$ aspirina, cotrimoxazol, hidrocodona-paracetamol (2,2 a 2,5\%) clopidogrel, cefalexina, penicilina, amoxicilina/clavulánico, azitromicina, levofloxacina, naproxeno, fenitoína, oxycodona/paracetamol, metformina (1 a 1,6\%).

\section{Conclusiones}

Los EAM que originaron visitas a CE constituyen una importante causa de morbilidad en los EE.UU., particularmente en mayores de 65 años. La vigilancia poblacional permanente puede ayudar a monitorizar estos eventos y contribuir al planeamiento de estrategias para su prevención.

Palabras Clave: Eventos adversos, vigilancia poblacional.

Key words: adverse effects, population surveillance.

Fuentes de financiamiento / Conflictos de interés: Centro de Control de Enfermedades (CDC) Administración de Drogas y Alimentos (FDA) Comisión de Seguridad de los Productos para los Consumidores de EE.UU.

\section{Comentario}

Los precios de los fármacos más vendidos en EEUU han superado dos a tres veces el rango de la inflación general ${ }^{1}$, creciendo el gasto de la prescripción para pacientes ambulatorios un promedio de $15 \%$ anual $^{2}$. En Argentina el crecimiento en ventas de medicamentos para el cuarto trimestre de 2006 también oscila en $15 \%{ }^{3}$.

El proyecto CADES parece ser una aproximación importante a la hora de registrar los EAM a nivel poblacional, tarea de por si compleja. Una herramienta de estas características garantiza la constancia de la cual carecen los sistemas de farmacovigilancia corrientes, basados en la notificación espontánea. Sin embargo, presenta como limitación que la evaluación de los datos se practique en forma retrospectiva, a través del relevamiento de las historias clínicas de las CE. De acuerdo a la gravedad del EAM, muchos pacientes no consultan ni resuelven allí sus inconvenientes con los medicamentos. Por otra parte, los profesionales de las centrales de emergencias, no siempre están entrenados para practicar farmacovigilancia.

El uso de medicamentos por pacientes ambulatorios viene en aumento por factores como: mayor número de población anciana, uso de farmacoterapia no sólo como tratamiento sino como quimioprofilaxis"; mayor disponibilidad de fármacos que cambian su condición de venta bajo receta a venta libre; propaganda de la industria farmacéutica dirigida directamente al paciente, enfermedades inventadas ("mongering diseases") y más facilidad para la adquisición (venta por Internet con envío a domicilio). Las responsabilidades para el paciente ambulatorio van entonces, desde la administración del medicamento, pasando por la adherencia al tratamiento y finalmente, su predisposición para notificar a los profesionales de la salud sobre la eficacia y la seguridad del tratamiento seguido, haya sido este por prescripción médica o autoadministrado ${ }^{5}$. Esto último hace que en el ámbito ambulatorio, no baste con un registro sistemático de consultas a CE, siendo aún necesaria la notificación espontánea de eventos adversos, tanto para fármacos conocidos como para los nuevos, sumado a medidas de profilaxis como educación para pacientes sobre el uso seguro de los medicamentos.

Gabriela Gonzalez Capdevila, Natalia Korzewycz y Roxana Beragua. [ Farmacéuticas, Área Farmacovigilancia, Hospital Italiano de Buenos Aires, gabriela.capdevila@ hospitalitaliano.org.ar ]

Capdevila G. Korzewycz N, Beragua R. Eventos adversos medicamentosos en pacientes ambulatorios. Evid. actual. práct. ambul; 10(5): 144, SepOct.2007. Comentado de: Budnitz D et al. National surveillance of emergency department visits for outpatient adverse drug events. JAMA 2006 Oct 18; 296:1858-1866. PMID: 17047216. Disponible en URL con suscripción:

http://www.ncbi.nlm.nih.gov/sites/entrez?Db=PubMed\&Cmd=ShowDetailView\&TermToSearch=17047216

Referencias

1. Hensley S. Drug prices rises as faster clip,placing burden on consumers. Wall Street Journal 2003 Apr 15;Sect D:4

2. Levit K. Health spending rebound continues in 2002. Health Aff 2004;23:147-59.

3. Instituto Nacional de Estadísticas y Censos (Indec). La industria farmacéutica en Argentina, cuarto trimestre de 2006 [en línea]. Información de prensa. 12 abril 2007. Disponible en URL: http://www.indec.gov.ar/nuevaweb/cuadros/13/farm_04_07.pdf (último acceso 18/10/07).

4.Anthony J. Brown, MD. Sildenafil Useful for SSRI-Related Sexual Dysfunction in Women. Reuters Health Information 2006. 2006 Reuters Ltd. Disponible en URL: http://www.medscape.com/viewarticle/533091 Último acceso: 4/09/2007.

5.Lam A. Use of Self-Prescribed Nonprescription Medications and Dietary Supplements Among Assisted Living Facility Residents. J Am Pharm Assoc. 2006;46(5):574-581. 